\title{
Immune Intervention in Type 1 Diabetes
}

\author{
Bimota Nambam ${ }^{1}$, Natasa Bratina ${ }^{2}$, and Desmond Schatz ${ }^{3}$
}

Introduction

M ULTIPLE STUDIES OVER THE PAST decade have reported residual $\beta$-cell function in early type 1 diabetes (T1D), which can last many years after diagnosis. Maintenance of residual endogenous insulin secretion is beneficial in achieving better glycemic control, and hence decreasing the risk of micro- and macrovascular complications. Although the etiopathogenesis of T1D remains to be determined, it is clear that derangements in the immune system play a vital role in the loss of tolerance and subsequent $\beta$-cell destruction. Several attempts have been made to interdict the disease at different stages of disease development. These include primary (before the development of islet autoantibodies or pre-stage 1 diabetes) and secondary prevention studies (islet autoantibody positive but before the development of clinical diabetes, or stage 1 and stage 2 diabetes) as well as in new-onset patients (stage 3 diabetes), and more recently, in patients with established diabetes. Although the disease has yet to be prevented or reversed, some studies have shown at least transient preservation of $\beta$-cell function. There is unlikely to be a complete cure with established clinical disease. However, with increasing rates of T1D, there will continue to be increased numbers of patients newly diagnosed with T1D providing opportunities for biologic interventions to alter the course of the disease. Primary prevention of T1D with a goal of preventing initiation of T1D autoimmunity still remains the "Holy Grail." A combination of therapies and approaches will become more of the future norm, together with enhanced understanding of the disease process. This article reviews some of the key immune interventional studies in at-risk and new-onset (stages 1-3) T1D that have been published (in print) between July 2016 and June 2017.

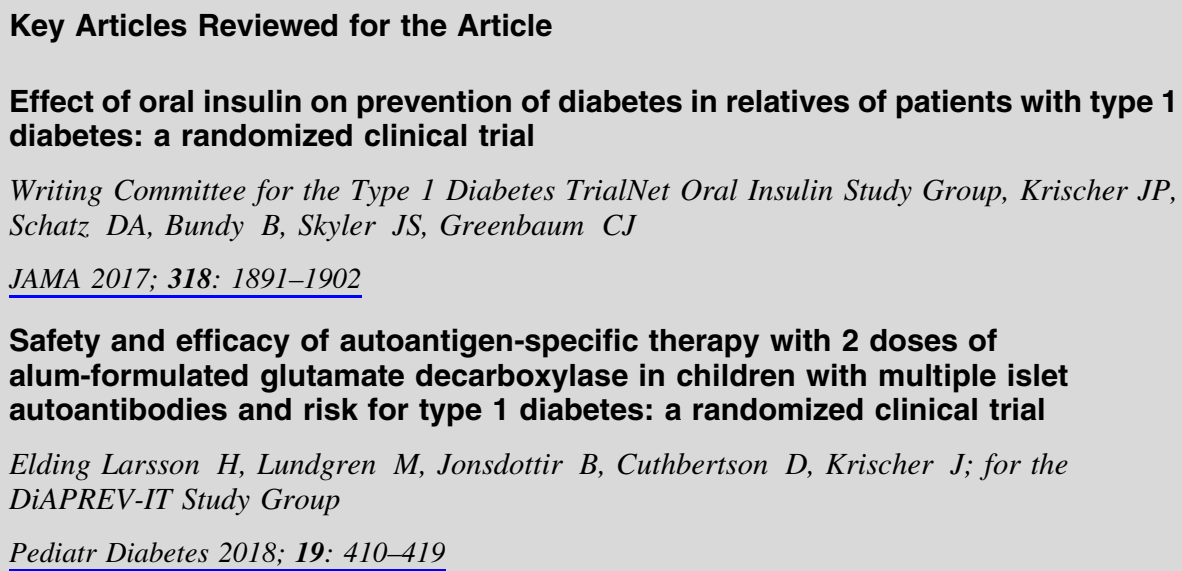

Elding Larsson H, Lundgren M, Jonsdottir B, Cuthbertson D, Krischer J; for the DiAPREV-IT Study Group

Pediatr Diabetes 2018; 19: 410-419

\footnotetext{
${ }^{1}$ Department of Pediatrics, Division of Endocrinology, Virginia Commonwealth University, Richmond, VA

${ }^{2}$ University Medical Centre, University Children's Hospital Ljubljana, Department of Endocrinology, Diabetes and Metabolic diseases, Ljubljana, Slovenia

${ }^{3}$ Department of Pediatrics, Division of Endocrinology, University of Florida, Gainesville, FL
} 


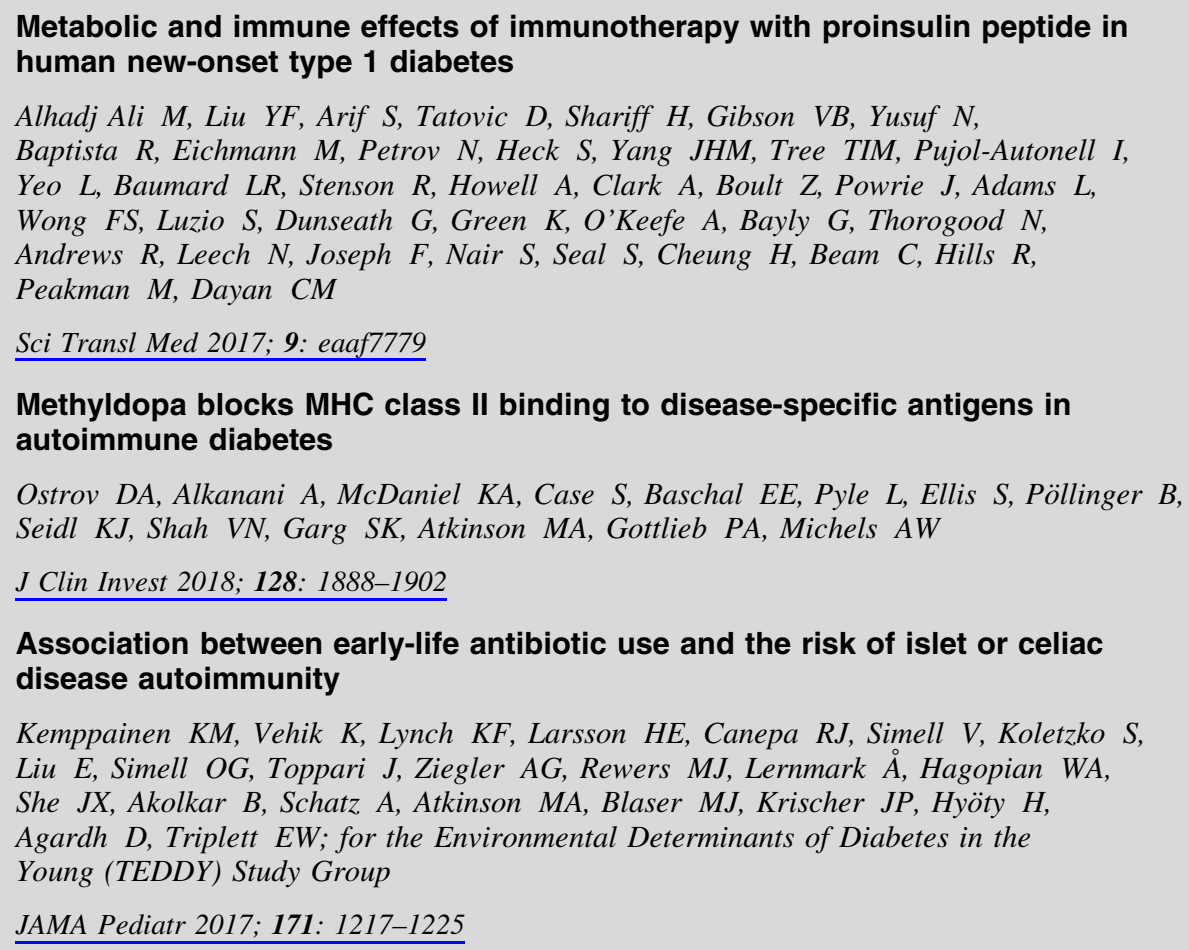

\section{Effect of oral insulin on prevention of diabetes in relatives of patients with type 1 diabetes: a randomized clinical trial}

Writing Committee for the Type 1 Diabetes TrialNet Oral Insulin Study Group, Krischer $J P^{l}$, Schatz $D A^{2}$, Bundy $B^{l}$, Skyler $\mathrm{JS}^{3}$, Greenbaum $\mathrm{CJ}^{4}$

${ }^{1}$ University of South Florida, Tampa, FL; ${ }^{2}$ University of Florida, Gainesville, FL; ${ }^{3}$ University of Miami, Miami, FL; ${ }^{4}$ Benaroya Research Institute, Seattle, WA

JAMA 2017; 318: 1891-1902

This manuscript is also discussed in the article on Diabetes Technology and Therapy in the Pediatric Age Group, page S-123.

\section{Background}

Greater understanding of the natural history of type 1 diabetes has occurred through the establishment of birth cohort studies (DIPP [Type 1 Diabetes Prediction and Prevention], BABYDIET, The Environmental Determinants of Diabetes in the Young [TEDDY] among others), as well as screening and monitoring of first- and second-degree relatives (TrialNet). The pursuit of safe preventative agents continues in the Diabetes Prevention Trial - Type 1 (DPT-1); oral insulin was shown in a post hoc analysis to delay the onset of the disease in at-risk subjects with higher insulin autoantibody titers. In order to fully determine whether oral insulin delayed progression of T1D autoimmunity to clinical disease, an appropriately powered study was conducted by TrialNet.

\section{Methods}

First-degree relatives (3-45 years of age) as well as second- and third-degree relatives (3-20 years) of T1D patients were enrolled in TrialNet. Patients with normal oral glucose tolerance and positive for microinsulin autoantibodies (mIAA) on two occasions, and negative for T1D protective human leukocyte antigen (HLA) haplotypes DQA $1 * 0102$ and $D Q B 1 * 0602$ were enrolled. The participants were also screened for glutamic acid decarboxylase (GAD) and insulinoma-associated antigen-2 (IA-2) autoantibodies and underwent an intravenous glucose tolerance test to assess first-phase insulin release (FPIR). The threshold for firstphase insulin was $\geq 60 \mu \mathrm{U} / \mathrm{mL}$ for participants 3-7 years old or parents of probands, and $\geq 100 \mu \mathrm{U} / \mathrm{mL}$ for siblings or offspring 8-45 years or other relatives 8-20 years of age. Eligible participants $(n=560)$ were randomized into treatment groups with oral insulin $(\mathrm{n}=276 ; 7.5 \mathrm{mg}$ daily) and placebo $(n=274)$. Randomization was stratified based on study site. The participants were divided into two strata, but these remained blinded until study completion. The primary stratum consisted of mIAA positive at-risk relatives who had positive ICA (islet cell antibodies) on two samples or GADA (GAD autoantibodies) and IA2 autoantibody positive on the same sample with confirmation of at least one of these autoantibodies on a separate sample, and first-phase insulin $>$ threshold. The mIAA positive secondary stratum was divided into three groups: Secondary stratum 1 subjects had a similar autoantibody profile as the primary stratum but with first-phase insulin less than threshold. In secondary stratum 2, ICA was negative or GADA/IA-2 autoantibodies were positive and confirmed on a separate sample. In this stratum, participants also had FPIR above the threshold. Secondary stratum 3 subjects were identical to secondary stratum 2, except participants had FPIR below the defined thresholds. The primary outcome was onset of diabetes in the primary stratum group as defined by the American Diabetes Association. 


\section{Results}

Follow-up time for the entire cohort was a median of 2.7 years. In the primary stratum, the annualized rate of diabetes was not significantly different between oral insulin $(8.8 \%)$ [95\% confidence interval (CI) $6.7 \%-11.2 \%$ ] and placebo (10.2\%) [95\% CI 7.8\%-12.9\%] with a hazard ratio (HR) of 0.87 [95\% CI $0-1.2 ; P=0.21]$. In the secondary stratum 1 , the annualized rate of diabetes was $18.1 \%$ [95\% CI 9.6\%-29.1\%] for the oral insulin group and $34.1 \%$ [95\% CI 20.6\%-51.1\%] for the placebo group (HR 0.45 [95\% CI 0-0.82]; $P=0.006$ ), while the annualized rate of diabetes for both the secondary 2 and 3 combined stratum was not different between oral insulin and controls $5.1 \%$ [95\% CI $2.6 \%-8.6 \%$ ] in the oral insulin group and $4.7 \%$ [95\% CI 2.2\%-8.0\%] for the placebo group (HR 1.03 [95\% CI 0-2.11]; $P=0.53$ ).

\section{Conclusion}

Oral insulin at a dose of $7.5 \mathrm{mg} /$ day did not delay or prevent the development of type 1 diabetes over 2.7 years in those with multiple autoantibodies compared with placebo.

\section{Comments}

In the initial DPT-1 study, $7.5 \mathrm{mg} /$ day of oral insulin failed to delay type 1 diabetes in at-risk subjects. However, an ad hoc analysis conducted in those with higher IAA titers demonstrated a delay in progression to disease. Those with higher IAA titers $(>80 \mathrm{nU} / \mathrm{mL})$ had an apparent 4- to 5-year delay in the onset of disease. This appropriately powered TrialNet study was conducted to confirm or negate these findings. Unfortunately, the overall study results were disappointing, albeit an effect was seen in a smaller number of subjects in the secondary stratum 1. This was surprising given that this stratum already had diminished FPIR yet still had a better response compared to the other strata. However, it is likely that oral insulin will not be effective once the disease has progressed (lost FPIR). Another reason for the ineffective outcome may be incorrect dosing. The $7.5 \mathrm{mg}$ dose investigated in this and the initial DPT study may not have been enough to elicit immunomodulatory effects. A higher dose $(67.5 \mathrm{mg}$ of oral insulin) was reported to produce protective insulin-responsive regulatory $\mathrm{T}$-cell responses in genetically at-risk young relatives (Pre-Point Study). TrialNet has recently completed enrollment of subjects building on these results and further investigating higher doses (67.5 mg daily vs $500 \mathrm{mg}$ every 2 weeks). A follow-up to the Pre-Point study (Pre-Point Early Study) is also being conducted where escalating doses of oral insulin $(7.5 \mathrm{mg} \times 3$ months followed by $22.5 \mathrm{mg} \times 3$ months, and then $67.5 \mathrm{mg} \times 6$ months) are being investigated in genetically at-risk children as young as 6 months to 2 years. Since T1D autoimmunity may occur as early as the first few years of life, oral insulin administration earlier may have a better chance of preventing initiation of autoimmunity. Even with the hope that these planned and ongoing studies would be successful and effects would be sustained, it is likely that multiple therapeutic strategies may be needed to produce clinically relevant effects.

\section{Safety and efficacy of autoantigen-specific therapy with 2 doses of alum-formulated glutamate decarboxylase in children with multiple islet autoantibodies and risk for type 1 diabetes: a randomized clinical trial}

Elding Larsson $H^{l}$, Lundgren $M^{l}$, Jonsdottir $B^{l}$, Cuthbertson $D^{2}$, Krischer $\mathrm{J}^{2}$; for the

DiAPREV-IT Study Group

${ }^{I}$ Department of Clinical Sciences/CRC, Skåne University Hospital, Lund University, Lund, Sweden; ${ }^{2}$ Health Informatics Institute, Morsani College of Medicine, University of South Florida, Tampa, FL

\section{Pediatr Diabetes 2018; 19: 410-419}

\section{Background}

Although administration of GAD antigen to NOD mice prevented autoimmune destruction of $\beta$-cells, human trials in new onset type 1 diabetes did not show efficacy (Diabetes Prevention Immune Tolerance). To determine whether earlier administration was effective, these investigators administered two doses of $20 \mu \mathrm{g}$ GAD-Alum in at-risk, multiple islet autoantibody-positive subjects in an attempt to prevent or delay the onset of clinical disease

\section{Methods}

The investigators conducted a randomized, placebo-controlled, double-blind study in nondiabetic children between the ages of 4 and 17.9 years with positive GADA autoantibodies and at least one of IA-2A, IAA, or ZnT8 autoantibodies. The major objectives of the study were to evaluate both the safety of GADAlum and potential efficacy in preventing or delaying progression to clinical type 1 diabetes. The eligible participants $(n=50)$ were randomized 1:1 to two doses of subcutaneous $20 \mu \mathrm{g}$ GADAlum or placebo. Further stratification was carried out according to the presence of two or three to six autoantibodies (three amino acid [R/W/Q] variants of ZnT8 considered as individual autoantibodies). Study participants were followed every 3 months for 5 years or until progression to clinical T1D.

\section{Results}

The administration of GAD-Alum did not affect time to clinical diagnosis. In addition, there was no difference in progression in those with two antibodies vs three to six antibodies (with the three ZnT8 variants taken as separate autoantibodies) There were no differences in glycated hemoglobin (HbA1c), first-phase insulin release or C-peptide area under the curve (AUC) between placebo and treated groups. There were no serious side effects.

\section{Conclusion}

Although GAD-Alum failed to prevent type 1 diabetes progression in those with multiple autoantibodies, it is safe in children and may warrant further study earlier in the disease or as combination therapy.

\section{Comments}

To date, antigen-based therapies have not been effective in preventing the disease. This may be due to multiple 
factors: (1) the therapy began too late in the disease process (participating children already had an impaired glucose tolerance test) or (2) incorrect dosing, dosing schedule, or route of administration (an ongoing study suggests intralymphatic injections may be effective). The authors have also hypothesized that GAD-Alum vaccine administered early in the course of T1D may be beneficial to those with DQ2 genotype, where patients present with GADA as the first antibody, as compared with DQ8 genotypes, where the first autoantibody to appear is IAA. Antigen-based therapies will likely be more successful when combined with other immunomodulatory therapies.

\section{Metabolic and immune effects of immunotherapy with proinsulin peptide in human new-onset type 1 diabetes}

Alhadj Ali $M^{1}$, Liu $Y F^{2,3}$, Arif $S^{2}$, Tatovic $D^{1}$, Shariff $H^{2}$, Gibson $V B^{2}$, Yusuf $N^{2}$, Baptista $R^{2,4}$, Eichmann $M^{2}$, Petrov $\mathrm{N}^{4}$, Heck $\mathrm{S}^{4}$, Yang $\mathrm{JHM}^{2}$, Tree $\mathrm{TIM}^{2}$, Pujol-Autonell $I^{2}$, Yeo L L ${ }^{2}$, Baumard $L R^{2}$, Stenson $R^{1}$, Howell $A^{1}$, Clark $A^{1}$, Boult $Z^{5}$, Powrie $J^{3}$, Adams $L^{3}$, Wong $F S^{1}$, Luzio $S^{6}$, Dunseath $G^{6}$, Green $K^{7}$, O'Keefe $A^{7}$, Bayly $G^{7}$, Thorogood $N^{7}$, Andrews $R^{7}$, Leech $N^{8}$, Joseph $F^{9}$, Nair $S^{9}$, Seal $S^{9}$, Cheung $H^{9}$, Beam $C^{10}$, Hills $R^{11}$, Peakman $M^{2,4,2}$, Dayan $C M^{1}$

${ }^{I}$ Diabetes Research Group, Cardiff University School of Medicine, Cardiff, UK; ${ }^{2}$ Department of Immunobiology, Faculty of Life Sciences and Medicine, King's College London, London, UK; ${ }^{3}$ Department of Diabetes and Endocrinology, Guy's and St Thomas' Hospital National Health Service (NHS) Foundation Trust, London, UK; ${ }^{4}$ National Institute of Health Research Biomedical Research Centre at Guy's and St Thomas' Hospital and King's College London, London, UK; ${ }^{5}$ Clinical Research Facility, University Hospital of Wales, Cardiff, UK; ${ }^{6}$ Diabetes Research Unit Cymru, Swansea University, Swansea, UK; ${ }^{7}$ Joint Clinical Research Unit, University Hospitals Bristol Foundation Trust, Bristol BS2 8HW, UK; ${ }^{8}$ Diabetes and Endocrinology Department, Newcastle upon Tyne Hospitals NHS Foundation Trust, Newcastle, UK; ${ }^{9}$ Diabetes and Endocrinology Department, Countess of Chester Hospital NHS Foundation Trust, Chester, UK; ${ }^{10}$ Division of Epidemiology and Biostatistics, Western Michigan University School of Medicine, MI; ${ }^{11}$ Haematology Clinical Trials Unit, Cardiff University School of Medicine, Cardiff, UK; ${ }^{12}$ King's Health Partners Institute of Diabetes, Endocrinology and Obesity, London, UK

Sci Transl Med 2017; 9: eaaf7779

\section{Background}

Given the heterogeneous nature of T1D, antigen-based therapies may be more successful in certain populations. The authors sought to investigate whether the administration of an HLA DR4-restricted, immunodominant region of proinsulin peptide would be safe and might modulate autoreactive CD4 $\mathrm{T}$ cells in new onset T1D patients.

\section{Methods}

The investigators conducted a randomized, doubleblinded, placebo-controlled phase 1b study. Enrolled subjects $(\mathrm{n}=27$ years, $18-45$ years, $<100$ days of diagnosis) were positive for HLA DRB $1 * 0401$, had one out of three autoantibodies positive (GAD, IA2, or ZnT8), and had a stimulated C-peptide on mixed-meal tolerance test of $>0.2 \mathrm{nM}$. Subjects were randomized to receive high-frequency (10$\mathrm{mg}$ proinsulin C19-A3 peptide every 2 weeks) or lowfrequency (10-mg proinsulin C19-A3 peptide every 4 weeks alternating with saline every 4 weeks) active treatment or placebo (saline injections every 2 weeks) by intradermal injection for a total of 12 administrations over 6 months. Residual C-peptide production was evaluated at baseline and then at 3, 6, 9, and 12 months. Autoreactive proinflammatory (IFN-gamma) and anti-inflammatory (IL-10) CD4 T-cell biomarkers of $\beta$-cell specific immune responses were also assessed at baseline, monthly, and 2 weeks after the last injection.

\section{Results}

There were no serious side effects related to peptide injection. The mean C-peptide over the duration of the study showed a significant decline from baseline in the placebo group at 3, 6, 9, and 12 months $(P=0.003, P=0.03, P=0.03$, $P=0.03$, respectively). A significant decline in mean Cpeptide was seen in the high-frequency group only at the 12month mark. IDAA1c (insulin dose adjusted A1c) values $(\mathrm{HbA} 1 \mathrm{c} \%+[4 \times$ insulin dose in units $/ \mathrm{kg} / \mathrm{day}]$ were significantly lower in the higher frequency arm at all time points, while it was significantly lower at 6 and 12 months in the lower frequency group compared to placebo. CD4 T-cell IL10 levels of C-peptide responders (post-baseline value more than $100 \%$ of baseline C-peptide AUC) to proinsulin stimulation were significantly higher in high- and low-frequency treatment groups compared with placebo $(P=0.0015$ and 0.003 , respectively).

\section{Conclusion}

Proinsulin peptide immunotherapy is safe, is associated with antigen-specific and nonspecific immune modulation, and may be efficacious in T1D.

\section{Comments}

Although this phase $1 \mathrm{~b}$ study was carried out in patients already diagnosed with T1D, given the increase in antiinflammatory responses of autoreactive CD4 T cells with administration of proinsulin peptide, its safety and application in those with already established autoimmunity but without T1D or those with high-risk genes but with still absent islet autoantibodies should be investigated. It is unlikely that this therapy will be highly beneficial in those with already established clinical disease. With the increase in the number of antigen-based trials and some promising outcomes, selection of a combination of safe agents that may be synergistic should be considered in future studies. 


\section{Methyldopa blocks MHC class II binding to disease- specific antigens in autoimmune diabetes}

Ostrov $D A^{1}$, Alkanani $A^{2,}$ McDaniel $K A^{2}$, Case $S^{2}$, Baschal EE ${ }^{2}$, Pyle $L^{2,3}$, Ellis $S^{2,4}$, Pöllinger $B^{5}$, Seidl $K^{5}$, Shah $V N^{2}$, Garg $S K^{2}$, Atkinson $M A^{1}$, Gottlieb $P A^{2}$, Michels $A W^{2}$

${ }^{1}$ Department of Pathology, Immunology, and Laboratory Medicine, College of Medicine, University of Florida, Gainesville, Florida; ${ }^{2}$ Barbara Davis Center for Childhood Diabetes, University of Colorado School of Medicine, Aurora, Colorado; ${ }^{3}$ Department of Biostatistics and Informatics, University of Colorado School of Public Health, Aurora, Colorado; ${ }^{4}$ Department of Clinical Pharmacy, University of Colorado, Aurora, Colorado; ${ }^{5}$ Novartis Institutes for Biomedical Research, Basel, Switzerland

J Clin Invest 2018; 128: 1888-1902

\section{Background}

The HLA-DQ8 allele (DQA1*03:01-DQB1*03:02) present in $50 \%$ to $60 \%$ of T1D patients provides an odds ratio for T1D disease development of 7 to 11. HLA class II genes (including the DQ8 allele) encode for major histocompatibility complex (MHC) class II proteins that are present on the surface of antigen presenting cells. MHC class II proteins form a trimolecular complex with an antigen peptide (including self-antigens), and the $\mathrm{T}$ cell receptor, and play a main role in autoimmunity. Organ donor studies from those with recent onset T1D have identified T cells restricted to proinsulin peptides presented by DQ8. The investigators hypothesized that blocking DQ8 antigen presentation might modify autoimmune response and represent a pathway for treating T1D.

\section{Methods}

Based on the crystalline structure of insulin B chain peptide (9-23 and 13-23) bound to DQ8 revealed by molecular docking techniques, different molecules were screened and those that occupied specific structural pockets (of DQ8) were identified and scored according to free energy of binding $(\Delta \mathrm{G})$. Based on the scores, these molecules were then tested for insulin/DQ8-specific $\mathrm{T}$ cell response in vitro. Further evaluation of the chemical structures of one of these topscoring compounds that occupied a key position in DQ8 antigen binding cleft revealed a tetraaza core chemical structure (tetraazatricylclododecane, TATD). A synthetically derived form of the TATD blocked critical interactions between T and B cells in NOD mice and prevented disease onset. Screening of almost 1200 Food and Drug Administration-approved drugs predicted to occupy this specific DQ8 pocket (P6) revealed methyl dopa as one of the drugs having a similar structure. Methyldopa was further evaluated in a single arm, open label, phase $1 \mathrm{~b}$ dose escalation human study in 20 DQ8 positive T1D patients between the ages of 18-46 years with disease duration less than 2 years, and with endogenous insulin production. All participants received methyldopa tablets orally of different doses (low dose: $500 \mathrm{mg} 2$ times/day, and moderate dose: $500 \mathrm{mg} 3$ times/day), while 18 of 20 tolerated the high dose ( $2-3 \mathrm{~g}$ selfadministered over the course of a day); the dosages were titrated over a 6-week period to prevent hypotension and allow for assessment of the ability of the different doses to block antigen presentation.

\section{Results}

Seventeen of the 20 participants showed significantly inhibited DQ8 restricted T cell responses compared with baseline during treatment phase with low, moderate, and high doses of methyldopa. The responses returned to baseline 6 weeks after cessation of therapy. DR4 and DQ2 responses were not altered. Two of the nonresponders were DQ8 homozygotes. There were no serious side effects of methyldopa. At 3 months, the C-peptide 2-hour AUC measurement following a mixedmeal tolerance test was similar to baseline levels.

\section{Conclusions}

Methyldopa treatment specifically inhibits DQ8 antigen presentation, but immune responses return to normal upon withdrawal of therapy. Blocking DQ8 antigen presentation may present a strategy to treat autoimmune diseases and other HLA-associated conditions.

\section{Comments}

This study highlights how current technological advances allowing minor manipulation of complex, specific immune activation pathways have the potential to change the course of autoimmune diseases. Even if the blocking was specific for DQ8 insulin restricted sites only and did not block antigen binding sites of other class MHCII molecules (DR4, DR3, DQ2), the authors hypothesized that given the phenomenon of epitope spreading in T1D, if DQ8 antigen binding sites were blocked early in the disease course, there could be even greater benefit. Although the study showed that only DQ8-restricted T cell responses to lower affinity selfantigens were inhibited and those to higher affinity remained intact, it remains unknown whether side effects may be seen long term. Somewhat analogous but opposite in nature to the commonly reported autoimmune side effects of checkpoint inhibitors, is there a risk of unwanted infections, and malignancies due to dampening of immune responses with this class of drugs? Nonetheless, this small but highly relevant study may lead to newer avenues in the current pursuit of prevention of T1D. Further studies in TrialNet are ongoing.

\section{Association between early-life antibiotic use and the risk of islet or celiac disease autoimmunity}

Kemppainen $K M^{1}$, Vehik $K^{2}$, Lynch $K F^{2}$, Larsson $H E^{3}$, Canepa $R^{1}$, Simell $V^{4}$, Koletzko $S^{5}$, Liu $E^{6}$, Simell $O^{7}{ }^{7}$, Toppari $J^{8,9}$, Ziegler $A G^{10,11,12}$, Rewers $M J^{13}$, Lernmark $\AA^{3}$, Hagopian $W^{14}$, She $J X^{15}$, Akolkar $B^{16}$, Schatz $D^{17}$, Atkinson $M A^{18}$, Blaser $\mathrm{MJ}^{19}$, Krischer $\mathrm{JP}^{2}$, Hyöty $\mathrm{H}^{20,21}$, Agardh $D^{3}$, Triplett $E W^{1}$; for the Environmental

Determinants of Diabetes in the Young (TEDDY) Study

Group

${ }^{I}$ Department of Microbiology and Cell Science, Institute of Food and Agricultural Sciences, University of Florida, 
Gainesville, FL; ${ }^{2}$ Health Informatics Institute, Morsani College of Medicine, University of South Florida, Tampa, FL; ${ }^{3}$ Department of Clinical Sciences, Lund University Clinical Research Center, Skåne University Hospital, Malmö, Sweden; ${ }^{4}$ MediCity Laboratory, University of Turku, Turku, Finland; ${ }^{5}$ Division of Paediatric Gastroenterology and Hepatology, Dr von Hauner Children's Hospital, Ludwig Maximilian University, München, Germany; ${ }^{6}$ Digestive Health Institute, Children's Hospital Colorado, Anschutz Medical Campus, University of Colorado Denver, Aurora, Co; ${ }^{7}$ Research Centre of Applied and Preventive Cardiovascular Medicine, University of Turku, Turku, Finland; ${ }^{8}$ Department of Pediatrics, University of Turku, Turku University Hospital, Turku, Finland; ${ }^{9}$ Department of Physiology, Institute of Biomedicine, University of Turku, Turku, Finland; ${ }^{10}$ Institute of Diabetes Research, Helmholtz Zentrum München, München, Germany; ${ }^{11}$ Klinikum Rechts der Isar, Technische Universität München, München, Germany; ${ }^{12}$ Forschergruppe Diabetes e.V., Neuherberg, Germany; ${ }^{13}$ Barbara Davis Center for Childhood Diabetes, University of Colorado Denver, Aurora, CO; ${ }^{14}$ Pacific Northwest Diabetes Research Institute, Seattle, WA; ${ }^{15}$ Center for Biotechnology and Genomic Medicine, Medical College of Georgia, Augusta University, Augusta, GA; ${ }^{16}$ National Institute of Diabetes and Digestive and Kidney Diseases, Bethesda, MD; ${ }^{17}$ Department of Pediatrics, College of Medicine, University of Florida, Gainesville, FL; ${ }^{18} \mathrm{De}$ partment of Pathology, Immunology, and Laboratory Medicine, College of Medicine, University of Florida, Gainesville, FL; ${ }^{19}$ Department of Medicine and Microbiology, New York School of Medicine, New York, NY; ${ }^{20}$ Department of Virology, Faculty of Medicine and Life Sciences, University of Tampere, Tampere, Finland; ${ }^{21}$ Fimlab Laboratories, Pirkanmaa Hospital District, Tampere, Finland

JAMA Pediatr 2017; 171: 1217-1225

\section{Background}

There has been increased use of antibiotics in the past few decades. It has been proposed that antibiotic use may be implicated in the growing incidence of autoimmune diseases including type 1 diabetes (T1D) and celiac disease (CD). Antibiotics administered to rodents predisposed to T1D may either protect or accelerate T1D development, especially if administered early in life, namely, in the prenatal and neonatal periods. The aim of this study was to test whether the use of antibiotics was associated with increased autoimmunity for T1D or CD in the first 4 years of life in The Environmental Determinants of Diabetes in the Young (TEDDY) cohort.

\section{Methods}

The TEDDY study is an international prospective cohort study that follows children with high genetic risk for T1D and/or CD at six clinical centers in four countries (Finland,
Germany, Sweden, and the United States). Infants were enrolled from the general population or among those having a first-degree relative with T1D if they had high-risk HLA alleles for T1D. Parents were asked about the use of the most commonly prescribed antibiotics (cephalosporins, penicillins, and macrolides) in their children between the age 3 months and 4 years. Data on antibiotic use during pregnancy was also collected. A total of 8495 children with a confirmed high-risk HLA genotype were screened for T1D autoantibodies four times per year up to the age of 4 years. They reached the age of 4 years in 2014 when analysis was done. Insulin autoantibodies (IAA), glutamic acid decarboxylase autoantibodies (GADA), and tyrosine phosphatase IA-2 autoantibodies (IA2A) were determined. Screening for CD started at the age of 12 months with measurement of tissue transglutaminase autoantibodies (tTGAs). The date of seroconversion was defined as the date of the first of two consecutive samples that tested positive for tTGAs.

\section{Results}

In the study group, antibiotics were prescribed 38,152 times. Amoxicillin was the most frequent antibiotic (42.3\%), with otitis media the most common cause for the use of antibiotics (62\%). Of the 8495 participants, 463 (5.5\%) developed islet autoantibodies before the age of 49.5 months, at a median age of 21.4 months. No association was found between antibiotic use soon after birth and just before seroconversion to islet autoantibodies. For CD, 783 of 6558 individuals (11.9\%) developed CD before the age of 49.5 months. CD seroconversion occurred at the median age of 30.6 months. Antibiotic use during the first or second year of life and within 6 months before the seroconversion period was not associated with CD. Maternal use of antibiotics during pregnancy was not associated with seroconversion for T1D or CD.

\section{Conclusion}

The present study showed that the use of antibiotics in early childhood is not associated with increased risk of T1D or $\mathrm{CD}$ in children with higher genetic risk.

\section{Comments}

Studies have implicated changes within the microbiome with the development of islet-related autoimmunity. How antibiotics influence such changes at a critical period of the development of the immune system needs to be further studied. The search for environmental determinants of T1D continues.

\section{Author Disclosure Statement}

No competing financial interests exist for all authors. 